\title{
The ascent-plateau statistics on Stirling permutations
}

\author{
Shi-Mei Ma* \\ School of Mathematics and Statistics \\ Northeastern University at Qinhuangdao \\ Hebei, P.R. China \\ shimeimapapers@163.com \\ Jun $\mathrm{Ma}^{\dagger}$ \\ Department of Mathematics \\ Shanghai Jiao Tong University \\ Shanghai, P.R. China \\ majun904@sjtu.edu.cn \\ Yeong-Nan $\mathrm{Yeh}^{\ddagger}$ \\ Institute of Mathematics \\ Academia Sinica \\ Taipei, Taiwan \\ mayeh@math.sinica.edu.tw
}

Submitted: Jul 17, 2018; Accepted: Mar 26, 2019; Published: Apr 5, 2019

(C) The authors. Released under the CC BY-ND license (International 4.0).

\begin{abstract}
A permutation $\sigma$ of the multiset $\{1,1,2,2, \ldots, n, n\}$ is called a Stirling permutation of order $n$ if $\sigma_{s}>\sigma_{i}$ as long as $\sigma_{i}=\sigma_{j}$ and $i<s<j$. In this paper, we present a unified refinement of the ascent polynomials and the ascent-plateau polynomials of Stirling permutations. In particular, by using Foata and Strehl's group action, we prove that the pairs of statistics (left ascent-plateau, ascent) and (left ascentplateau, plateau) are equidistributed over Stirling permutations of given order, and we show the $\gamma$-positivity of the enumerative polynomial of left ascent-plateaus, double ascents and descent-plateaus. A connection between the $\gamma$-coefficients of this enumerative polynomial and Eulerian numbers is also established.
\end{abstract}

Mathematics Subject Classifications: 05A05, 05A15

\section{Introduction}

A Stirling permutation of order $n$ is a permutation of the multiset $\{1,1,2,2, \ldots, n, n\}$ such that for each $i, 1 \leqslant i \leqslant n$, all entries between the two occurrences of $i$ are larger than $i$.

Denote by $\mathcal{Q}_{n}$ the set of Stirling permutations of order $n$. Let $\sigma=\sigma_{1} \sigma_{2} \cdots \sigma_{2 n} \in \mathcal{Q}_{n}$. For $1 \leqslant i \leqslant 2 n$, we say that an index $i$ is a descent of $\sigma$ if $\sigma_{i}>\sigma_{i+1}$ or $i=2 n$, and we say

${ }^{*}$ Supported by Natural Science Foundation of Hebei Province (A2017501007) and NSFC (11401083).

${ }^{\dagger}$ Supported by NSFC 11571235 .

${ }_{\ddagger}^{\ddagger}$ Supported by NSC 107-2115-M-001-009-MY3. 
that an index $i$ is an ascent of $\sigma$ if $\sigma_{i-1}<\sigma_{i}$ or $i=1$. Hence the index $i=1$ is always an ascent and $i=2 n$ is always a descent. A plateau of $\sigma$ is an index $i$ such that $\sigma_{i}=\sigma_{i+1}$, where $1 \leqslant i \leqslant 2 n-1$. Let $\operatorname{des}(\sigma)$, asc $(\sigma)$ and plat $(\sigma)$ denote the numbers of descents, ascents and plateaus of $\sigma$, respectively.

Stirling permutations were introduced by Gessel and Stanley [7], and they proved that

$$
(1-x)^{2 k+1} \sum_{n=0}^{\infty}\left\{\begin{array}{c}
n+k \\
n
\end{array}\right\} x^{n}=\sum_{\sigma \in \mathcal{Q}_{k}} x^{\operatorname{des} \sigma},
$$

where $\left\{\begin{array}{c}n \\ k\end{array}\right\}$ is the Stirling number of the second kind, i.e., the number of ways to partition a set of $n$ objects into $k$ non-empty subsets. A classical result of Bóna [2] says that descents, ascents and plateaus are equidistributed, i.e.,

$$
\sum_{\sigma \in \mathcal{Q}_{n}} x^{\operatorname{des} \sigma}=\sum_{\sigma \in \mathcal{Q}_{n}} x^{\text {asc } \sigma}=\sum_{\sigma \in \mathcal{Q}_{n}} x^{\text {plat } \sigma} .
$$

This equidistributed result have been extensively studied by Janson, Kuba, Panholzer, Haglund, Visontai, Chen, Fu et al., see [5, 8, 9, 10] and references therein.

It is natural to explore multivariate extension of (1). Let us now recall some definitions.

Definition 1 ([14]). An occurrence of an ascent-plateau of $\sigma \in \mathcal{Q}_{n}$ is an index $i$ such that $\sigma_{i-1}<\sigma_{i}=\sigma_{i+1}$, where $i \in\{2,3, \ldots, 2 n-1\}$. An occurrence of a left ascent-plateau is an index $i$ such that $\sigma_{i-1}<\sigma_{i}=\sigma_{i+1}$, where $i \in\{1,2, \ldots, 2 n-1\}$ and $\sigma_{0}=0$.

Let ap $(\sigma)$ and lap $(\sigma)$ be the numbers of ascent-plateaus and left ascent-plateaus of $\sigma$, respectively. For example, ap $(442332115665)=2$ and lap $(442332115665)=3$.

Define

$$
\begin{aligned}
& M_{n}(x)=\sum_{\sigma \in \mathcal{Q}_{n}} x^{\mathrm{ap}(\sigma)}, \\
& N_{n}(x)=\sum_{\sigma \in \mathcal{Q}_{n}} x^{\operatorname{lap}(\sigma)} .
\end{aligned}
$$

According to [14, Theorem 2, Theorem 3], we have

$$
\begin{aligned}
& M(x, t)=\sum_{n \geqslant 0} M_{n}(x) \frac{t^{n}}{n !}=\sqrt{\frac{x-1}{x-e^{2 t(x-1)}}}, \\
& N(x, t)=\sum_{n \geqslant 0} N_{n}(x) \frac{t^{n}}{n !}=\sqrt{\frac{1-x}{1-x e^{2 t(1-x)}}} .
\end{aligned}
$$

Clearly, $M_{n}(x)=x^{n} N_{n}\left(\frac{1}{x}\right)$. The reader is referred to $[16,17]$ for further properties of these polynomials.

Let

$$
C_{n}(x)=\sum_{\sigma \in \mathcal{Q}_{n}} x^{\mathrm{asc}(\sigma)}
$$


The polynomials $C_{n}(x)$ and $N_{n}(x)$, respectively, satisfy the following recurrence relation

$$
\begin{gathered}
C_{n+1}(x)=(2 n+1) x C_{n}(x)+x(1-x) C_{n}^{\prime}(x), \\
N_{n+1}(x)=(2 n+1) x N_{n}(x)+2 x(1-x) N_{n}^{\prime}(x),
\end{gathered}
$$

with the initial conditions $C_{0}(x)=N_{0}(x)=1$ (see $[2,7,13]$ for instance). The purpose of this paper is to present a unified refinement of the polynomials $C_{n}(x)$ and $N_{n}(x)$. In the sequel, we always assume that Stirling permutations are prepended by 0 . That is, we identify an $n$-Stirling permutation $\sigma_{1} \sigma_{2} \cdots \sigma_{2 n}$ with the word $\sigma_{0} \sigma_{1} \sigma_{2} \cdots \sigma_{2 n}$, where $\sigma_{0}=0$.

In this paper, we introduce the following definition.

Definition 2. Let $\sigma=\sigma_{1} \sigma_{2} \cdots \sigma_{2 n} \in \mathcal{Q}_{n}$. For $1 \leqslant i \leqslant 2 n-1$, a double ascent of $\sigma$ is an index $i$ such that $\sigma_{i-1}<\sigma_{i}<\sigma_{i+1}$, a descent-plateau of $\sigma$ is an index $i$ such that $\sigma_{i-1}>\sigma_{i}=\sigma_{i+1}$.

Let dasc $(\sigma)$ and $\operatorname{dp}(\sigma)$ denote the numbers of double ascents and descent-plateaus of $\sigma$, respectively. For example, dasc $(\mathbf{2 4 4 3 3 2 1 1 5 6 6 5 )}=2$ and $\operatorname{dp}(244332115665)=2$. It is clear that

$$
\operatorname{asc}(\sigma)=\operatorname{lap}(\sigma)+\operatorname{dasc}(\sigma), \operatorname{plat}(\sigma)=\operatorname{lap}(\sigma)+\operatorname{dp}(\sigma) .
$$

It is natural to consider the polynomials $P_{n}(x, y, z)$ defined by

$$
P_{n}(x, y, z)=\sum_{\sigma \in \mathcal{Q}_{n}} x^{\mathrm{lap}(\sigma)} y^{\mathrm{dasc}(\sigma)} z^{\mathrm{dp}(\sigma)}=\sum_{i, j, k} p_{n}(i, j, k) x^{i} y^{j} z^{k}
$$

where $1 \leqslant i \leqslant n, 0 \leqslant j \leqslant n-1,0 \leqslant k \leqslant n-1$. In particular,

$$
\begin{gathered}
P_{n}(x, x, 1)=P_{n}(x, 1, x)=C_{n}(x), \\
P_{n}(x, 1,1)=N_{n}(x) .
\end{gathered}
$$

As a continuation of [2], the main result of this paper says that the pairs of statistics (lap $(\sigma)$, asc $(\sigma))$ and (lap $(\sigma)$, plat $(\sigma))$ are equidistributed. The main tools of the proofs are the grammatical technique and a variation of the Foata and Strehl's group action.

\section{Main results}

Context-free grammars can be used to study various exponential structures (see [4, $5,15,17]$ for instance). For an alphabet $A$, let $\mathbb{Q}((A))$ be the ring of formal Laurent series formed from letters in $A$. Following [4], a context-free grammar over $A$ is a function $G: A \rightarrow \mathbb{Q}((A))$ that replaces a letter in $A$ by an element of $\mathbb{Q}((A))$. The formal derivative $D$ is a linear operator defined with respect to a context-free grammar $G$. According to [5], an advantage of the grammatical description of a combinatorial sequence is that a recursion of its generating function can be provided by attaching a labelling of the combinatorial object in accordance with the replacement rules of the grammar. 


\subsection{A grammatical labeling of Stirling permutations}

The first few of the polynomials $P_{n}(x, y, z)$ are given as follows:

$$
\begin{aligned}
& P_{1}(x, y, z)=x \\
& P_{2}(x, y, z)=x y+x z+x^{2}, \\
& P_{3}(x, y, z)=x\left(y^{2}+z^{2}\right)+4 x^{2}(y+z)+2 x y z+2 x^{2}+x^{3} .
\end{aligned}
$$

Theorem 3. Let $A=\{x, y, z, p, q\}$ and

$$
G=\{x \rightarrow x z q, y \rightarrow y z p, z \rightarrow x y z, p \rightarrow x y z, q \rightarrow x y z\} .
$$

Then

$$
D^{n}(z)=z \sum_{i, j, k} p_{n}(i, j, k)(x y)^{i} q^{j} p^{k} z^{2 n-2 i-j-k},
$$

where $1 \leqslant i \leqslant n, 0 \leqslant j \leqslant n-1,0 \leqslant k \leqslant n-1$ and $2 i+j+k \leqslant 2 n$. Set $P_{n}=P_{n}(x, y, z)$. Then the polynomials $P_{n}(x, y, z)$ satisfy the recurrence relation

$$
P_{n+1}=(2 n+1) x P_{n}+\left(x y+x z-2 x^{2}\right) \frac{\partial}{\partial x} P_{n}+x(1-y) \frac{\partial}{\partial y} P_{n}+x(1-z) \frac{\partial}{\partial z} P_{n},
$$

with the initial condition $P_{0}(x, y, z)=1$.

Proof. Now we give a labeling of $\sigma \in \mathcal{Q}_{n}$ as follows:

$\left(L_{1}\right)$ If $i$ is a left ascent-plateau, then put a superscript label $y$ immediately before $\sigma_{i}$ and a superscript label $x$ right after $\sigma_{i}$;

$\left(L_{2}\right)$ If $i$ is a double ascent, then put a superscript label $q$ immediately before $\sigma_{i}$;

$\left(L_{3}\right)$ If $i$ is a descent-plateau, then put a superscript label $p$ right after $\sigma_{i}$;

$\left(L_{4}\right)$ The rest positions in $\sigma$ are labeled by superscript labels $z$. It should be noted that the labels $z$ mark the descent positions of $\sigma$.

The weight of $\sigma$ is defined by

$$
w(\sigma)=z(x y)^{\operatorname{lap}(\sigma)} q^{\operatorname{dasc}(\sigma)} p^{\operatorname{dp}(\sigma)} z^{2 n-2 \operatorname{lap}(\sigma)-\operatorname{dasc}(\sigma)-\operatorname{dp}(\sigma)} .
$$

For example, the labeling of 552442998813316776 is as follows:

$$
{ }^{y} 5^{x} 5^{z} 2^{y} 4^{x} 4^{z} 2^{y} 9^{x} 9^{z} 8^{p} 8^{z} 1^{y} 3^{x} 3^{z} 1^{q} 6^{y} 7^{x} 7^{z} 6^{z} .
$$

We proceed by induction on $n$. Note that $\mathcal{Q}_{1}=\left\{{ }^{y} 1^{x} 1^{z}\right\}$ and

$$
\mathcal{Q}_{2}=\left\{{ }^{y} 1^{x} 1^{y} 2^{x} 2^{z},{ }^{q} 1^{y} 2^{x} 2^{z} 1^{z},{ }^{y} 2^{x} 2^{z} 1^{p} 1^{z}\right\} .
$$

Thus the weight of ${ }^{y} 1^{x} 1^{z}$ is given by $D(z)$ and the sum of weights of elements in $\mathcal{Q}_{2}$ is given by $D^{2}(z)$, since $D(z)=x y z$ and $D^{2}(x)=z\left(x y q z+x y p z+x^{2} y^{2}\right)$.

Assume that the result holds for $n=m-1$, where $m \geqslant 3$. Let $\sigma$ be an element counted by $p_{m-1}(i, j, k)$, and let $\sigma^{\prime}$ be an element of $\mathcal{Q}_{m}$ obtained by inserting the pair $m m$ into $\sigma$. We distinguish five cases: 
$\left(c_{1}\right)$ If the pair $m m$ is inserted at a position with label $x$, then the change of labeling is illustrated as follows:

$$
\cdots \sigma_{\ell-1}^{y} \sigma_{\ell}^{x} \sigma_{\ell+1} \cdots \mapsto \cdots \sigma_{\ell-1}^{q} \sigma_{\ell}^{y} m^{x} m^{z} \sigma_{\ell+1} \cdots
$$

In this case, the insertion corresponds to the rule $x \mapsto x z q$ and produces $i$ permutations in $\mathcal{Q}_{m}$ with $i$ left ascent-plateaus, $j+1$ double ascents and $k$ descent-plateaus;

$\left(c_{2}\right)$ If the pair $m m$ is inserted at a position with label $y$, then the change of labeling is illustrated as follows:

$$
\cdots \sigma_{\ell-1}^{y} \sigma_{\ell}^{x} \sigma_{\ell+1} \cdots \mapsto \cdots \sigma_{\ell-1}^{y} m^{x} m^{z} \sigma_{\ell}^{p} \sigma_{\ell+1} \cdots .
$$

In this case, the insertion corresponds to the rule $y \mapsto y z p$ and produces $i$ permutations in $\mathcal{Q}_{m}$ with $i$ left ascent-plateaus, $j$ double ascents and $k+1$ descent-plateaus;

$\left(c_{3}\right)$ If the pair $m m$ is inserted at a position with label $z$, then the change of labeling is illustrated as follows:

$$
\cdots \sigma_{\ell}^{z} \sigma_{\ell+1} \cdots \mapsto \cdots \sigma_{\ell}^{y} m^{x} m^{z} \sigma_{\ell+1} \cdots
$$

In this case, the insertion corresponds to the rule $z \mapsto x y z$ and produces $2 m-2-$ $2 i-j-k$ permutations in $\mathcal{Q}_{m}$ with $i+1$ left ascent-plateaus, $j$ double ascents and $k$ descent-plateaus;

$\left(c_{4}\right)$ If the pair $m m$ is inserted at a position with label $q$, then the change of labeling is illustrated as follows:

$$
\cdots \sigma_{\ell}^{q} \sigma_{\ell+1} \cdots \mapsto \cdots \sigma_{\ell}^{y} m^{x} m^{z} \sigma_{\ell+1} \cdots
$$

In this case, the insertion corresponds to the rule $q \mapsto x y z$ and produces $j$ permutations in $\mathcal{Q}_{m}$ with $i+1$ left ascent-plateaus, $j-1$ double ascents and $k$ descentplateaus;

$\left(c_{5}\right)$ If the pair $m m$ is inserted at a position with label $p$, then the change of labeling is illustrated as follows:

$$
\cdots \sigma_{\ell}^{p} \sigma_{\ell+1} \cdots \mapsto \cdots \sigma_{\ell}^{y} m^{x} m^{z} \sigma_{\ell+1} \cdots
$$

In this case, the insertion corresponds to the rule $p \mapsto x y z$ and produces $k$ permutations in $\mathcal{Q}_{m}$ with $i+1$ left ascent-plateaus, $j$ double ascents and $k-1$ descentplateaus.

By induction, we see that grammar (3) generates all elements in $\mathcal{Q}_{m}$. Combining the above five cases, we see that

$$
\begin{aligned}
p_{n+1}(i, j, k)= & i p_{n}(i, j-1, k)+i p_{n}(i, j, k-1)+(j+1) p_{n}(i-1, j+1, k)+ \\
& (k+1) p_{n}(i-1, j, k+1)+(2 n+3-2 i-j-k) p_{n}(i-1, j, k) .
\end{aligned}
$$

Multiplying both sides of the above recurrence relation by $x^{i} y^{j} z^{k}$ for all $i, j, k$, we get (4). 


\subsection{Equidistributed statistics}

Let $i \in[2 n]$ and let $\sigma=\sigma_{1} \sigma_{2} \ldots \sigma_{2 n} \in \mathcal{Q}_{n}$. We define an action $\varphi_{i}$ on $\mathcal{Q}_{n}$ as follows:

- If $i$ is a double ascent, then $\varphi_{i}(\sigma)$ is obtained by moving $\sigma_{i}$ to the right of the second $\sigma_{i}$, which forms a new pleateau $\sigma_{i} \sigma_{i}$;

- If $i$ is a descent-plateau, then $\varphi_{i}(\sigma)$ is obtained by moving $\sigma_{i}$ to the right of $\sigma_{k}$, where $k=\max \left\{j \in\{0,1,2, \ldots, i-1\}: \sigma_{j}<\sigma_{i}\right\}$.

For instance, if $\sigma=2447887332115665$, then

$$
\varphi_{1}(\sigma)=4478873322115665, \varphi_{4}(\sigma)=2448877332115665,
$$

and $\varphi_{9}\left(\varphi_{1}(\sigma)\right)=\varphi_{6}\left(\varphi_{4}(\sigma)\right)=\sigma$. In recent years, the Foata and Strehl's group actions have been extensively studied (see $[3,11]$ for instance). We introduce the Foata-Strehl action on Stirling permutations by

$$
\varphi_{i}^{\prime}(\sigma)= \begin{cases}\varphi_{i}(\sigma), & \text { if } i \text { is a double ascent or descent-plateau } \\ \sigma, & \text { otherwise. }\end{cases}
$$

It is clear that $\varphi_{i}^{\prime}$ are involutions and that they commute. Hence, for any subset $S \subseteq[2 n]$, we may define the function $\varphi_{S}^{\prime}: \mathcal{Q}_{n} \mapsto \mathcal{Q}_{n}$ by $\varphi_{S}^{\prime}(\sigma)=\prod_{i \in S} \varphi_{i}^{\prime}(\sigma)$. Then the group $\mathbb{Z}_{2}^{2 n}$ acts on $\mathcal{Q}_{n}$ via the function $\varphi_{S}^{\prime}$, where $S \subseteq[2 n]$.

The main result of this paper is given as follows, which is also implied by (4).

Theorem 4. For any $n \geqslant 1$, we have

$$
P_{n}(x, y, z)=P_{n}(x, z, y)
$$

Furthermore,

$$
\sum_{\sigma \in \mathcal{Q}_{n}} x^{\operatorname{lap}(\sigma)} y^{\operatorname{asc}(\sigma)}=\sum_{\sigma \in \mathcal{Q}_{n}} x^{\operatorname{lap}(\sigma)} y^{\text {plat }(\sigma)}
$$

Proof. For any $\sigma \in \mathcal{Q}_{n}$, we define

$$
\begin{aligned}
\operatorname{Dasc}(\sigma) & =\left\{i \in[2 n-1]: \sigma_{i-1}<\sigma_{i}<\sigma_{i+1}\right\}, \\
\operatorname{DP}(\sigma) & =\left\{i \in[2 n-1]: \sigma_{i-1}>\sigma_{i}=\sigma_{i+1}\right\}, \\
\operatorname{LAP}(\sigma) & =\left\{i \in[2 n-1]: \sigma_{i-1}<\sigma_{i}=\sigma_{i+1}\right\} .
\end{aligned}
$$

Let $S=S(\sigma)=\operatorname{Dasc}(\sigma) \cup \operatorname{DP}(\sigma)$. Note that

$$
\operatorname{Dasc}\left(\varphi_{S}^{\prime}(\sigma)\right)=\operatorname{DP}(\sigma), \operatorname{DP}\left(\varphi_{S}^{\prime}(\sigma)\right)=\operatorname{Dasc}(\sigma) \text { and } \operatorname{LAP}\left(\varphi_{S}^{\prime}(\sigma)\right)=\operatorname{LAP}(\sigma) .
$$

Therefore,

$$
P_{n}(x, y, z)=\sum_{\sigma \in \mathcal{Q}_{n}} x^{\operatorname{lap}(\sigma)} y^{\operatorname{dasc}(\sigma)} z^{\mathrm{dp}(\sigma)}
$$




$$
\begin{aligned}
& =\sum_{\sigma \in \mathcal{Q}_{n}} x^{\operatorname{lap}\left(\varphi_{S}^{\prime}(\sigma)\right)} y^{\mathrm{dp}\left(\varphi_{S}^{\prime}(\sigma)\right)} z^{\operatorname{dasc}\left(\varphi_{S}^{\prime}(\sigma)\right)} \\
& =\sum_{\sigma^{\prime} \in \mathcal{Q}_{n}} x^{\operatorname{lap}\left(\sigma^{\prime}\right)} z^{\operatorname{dasc}\left(\sigma^{\prime}\right)} y^{\operatorname{dp}\left(\sigma^{\prime}\right)} \\
& =P_{n}(x, z, y) .
\end{aligned}
$$

Combining (2) and (5), we see that $P_{n}(x y, y, 1)=P_{n}(x y, 1, y)$. This completes the proof.

Theorem 5. For $n \geqslant 1$, we have

$$
\sum_{\sigma \in \mathcal{Q}_{n}} x^{\mathrm{lap}(\sigma)} y^{\mathrm{dasc}(\sigma)} z^{\mathrm{dp}(\sigma)}=\sum_{\substack{1 \leqslant i \leqslant n \\ 0 \leqslant j \leqslant n-1}} \gamma_{n, i, j} x^{i}(y+z)^{j},
$$

where

$$
\gamma_{n, i, j}=\#\left\{\sigma \in \mathcal{Q}_{n}: \operatorname{lap}(\sigma)=i, \operatorname{dasc}(\sigma)=j, \operatorname{dp}(\sigma)=0\right\} .
$$

Proof. Let $\mathrm{NDP}_{n}=\left\{\sigma \in \mathcal{Q}_{n}: \operatorname{dp}(\sigma)=0\right\}$, and let

$$
\mathrm{NDP}_{n, i, j}=\left\{\sigma \in \mathcal{Q}_{n}: \operatorname{lap}(\sigma)=i, \operatorname{dasc}(\sigma)=j, \operatorname{dp}(\sigma)=0\right\} .
$$

For any $\sigma \in \mathrm{NDP}_{n, i, j}$, let $[\sigma]=\left\{\varphi_{S}^{\prime}(\sigma) \mid S \subseteq \operatorname{Dasc}(\sigma)\right\}$. For any $\sigma^{\prime} \in[\sigma]$, suppose that $\sigma^{\prime}=\varphi_{S}^{\prime}(\sigma)$ for some $S \subseteq \operatorname{Dasc}(\sigma)$. Then

$$
\operatorname{lap}\left(\sigma^{\prime}\right)=\operatorname{lap}(\sigma), \operatorname{dasc}\left(\sigma^{\prime}\right)=\operatorname{dasc}(\sigma)-|S| \text { and } \operatorname{dp}\left(\sigma^{\prime}\right)=|S| .
$$

Moreover, $\left\{[\sigma] \mid \sigma \in \operatorname{NDP}_{n}\right\}$ form a partition of $\mathcal{Q}_{n}$. Hence,

$$
\begin{aligned}
& \sum_{\sigma \in \mathcal{Q}_{n}} x^{\operatorname{lap}(\sigma)} y^{\operatorname{dasc}(\sigma)} z^{\mathrm{dp}(\sigma)} \\
= & \sum_{\sigma \in \mathrm{NDP}_{n}} \sum_{\sigma^{\prime} \in[\sigma]} x^{\operatorname{lap}\left(\sigma^{\prime}\right)} y^{\operatorname{dasc}\left(\sigma^{\prime}\right)} z^{\mathrm{dp}\left(\sigma^{\prime}\right)} \\
= & \sum_{\sigma \in \mathrm{NDP}_{n}} \sum_{S \subseteq \operatorname{Dasc}(\sigma)} x^{\operatorname{lap}\left(\varphi_{S}^{\prime}(\sigma)\right)} y^{\operatorname{dasc}\left(\varphi_{S}^{\prime}(\sigma)\right)} z^{\mathrm{dp}\left(\varphi_{S}^{\prime}(\sigma)\right)} \\
= & \sum_{\sigma \in \mathrm{NDP}_{n}} \sum_{S \subseteq \operatorname{Dasc}(\sigma)} x^{\operatorname{lap}(\sigma)} y^{\operatorname{dasc}(\sigma)-|S|} z^{|S|} \\
= & \sum_{\sigma \in \mathrm{NDP}_{n}} x^{\operatorname{lap}(\sigma)} \sum_{S \subseteq \operatorname{Dasc}(\sigma)} y^{\operatorname{dasc}(\sigma)-|S|} z^{|S|} \\
= & \sum_{\sigma \in \mathrm{NDP}_{n}} x^{\operatorname{lap}(\sigma)}(y+z)^{\operatorname{dasc}(\sigma)} \\
= & \sum_{i, j} \gamma_{n, i, j} x^{i}(y+z)^{j} .
\end{aligned}
$$


Taking $y=z=1$ in Theorem 5 , we have

$$
N_{n}(x)=\sum_{\sigma \in \mathcal{Q}_{n}} x^{\operatorname{lap}(\sigma)}=\sum_{i=1}^{n}\left(\sum_{j=0}^{n-1} 2^{j} \gamma_{n, i, j}\right) x^{i} .
$$

Let $N_{n}(x)=\sum_{k=1}^{n} N(n, k) x^{k}$. According to [13, Eq. (24)], we have

$$
N_{n}(x)=\sum_{k=1}^{n} 2^{n-2 k}\left(\begin{array}{c}
2 k \\
k
\end{array}\right) k !\left\{\begin{array}{l}
n \\
k
\end{array}\right\} x^{k}(1-x)^{n-k} .
$$

Thus, for $n \geqslant 1$, we have

$$
\sum_{j=0}^{n-1} 2^{j} \gamma_{n, i, j}=\sum_{j=1}^{i}(-1)^{i-j} 2^{n-2 j}\left(\begin{array}{c}
2 j \\
j
\end{array}\right)\left(\begin{array}{c}
n-j \\
i-j
\end{array}\right) j !\left\{\begin{array}{l}
n \\
j
\end{array}\right\} .
$$

Theorem 6. Let $A=\{u, v, w\}$ and $G=\{u \rightarrow u v w, v \rightarrow 2 u w, w \rightarrow u w\}$. Then

$$
D^{n}(w)=\sum_{\substack{1 \leqslant i \leqslant n \\ 0 \leqslant j \leqslant n-1}} \gamma_{n, i, j} u^{i} v^{j} w^{2 n+1-2 i-j} .
$$

Furthermore, the numbers $\gamma_{n, i, j}$ satisfy the recurrence relation

$$
\gamma_{n+1, i, j}=i \gamma_{n, i, j-1}+2(j+1) \gamma_{n, i-1, j+1}+(2 n+3-2 i-j) \gamma_{n, i-1, j},
$$

with the initial conditions $\gamma_{1,1,0}=1$ and $\gamma_{1, i, j}=0$ for $i>1$ and $j \geqslant 0$.

Proof. From the grammar (3), we see that

$$
\begin{aligned}
D(x y) & =x y z(p+q), \\
D(p+q) & =2 x y z, \\
D(z) & =x y z .
\end{aligned}
$$

Set $u=x y, v=p+q$ and $w=z$. Then $D(u)=u v w, D(v)=2 u w$ and $D(w)=u w$. Combining Theorem 3 and Theorem 5 , we get (7). Since $D^{n+1}(w)=D\left(D^{n}(w)\right)$, we obtain that

$$
\begin{aligned}
D^{n+1}(w) & =D\left(\sum_{i, j} \gamma_{n, i, j} u^{i} v^{j} w^{2 n+1-2 i-j}\right) \\
& =\sum_{i, j} i \gamma_{n, i, j} u^{i} v^{j+1} w^{2 n+2-2 i-j}+2 \sum_{i, j} j \gamma_{n, i, j} u^{i+1} v^{j-1} w^{2 n+2-2 i-j}+ \\
& \sum_{i, j}(2 n+1-2 i-j) \gamma_{n, i, j} u^{i+1} v^{j} w^{2 n+1-2 i-j}
\end{aligned}
$$

Equating the coefficients of $u^{i} v^{j} w^{2 n+1-2 i-j}$ on both sides of the above equation, we obtain (8). 
Let $G_{n}(x, y)=\sum_{i, j} \gamma_{n, i, j} x^{i} y^{j}$. Multiplying both sides of the recurrence relation (8) by $x^{i} y^{j}$ for all $i, j$, we get that

$$
G_{n+1}(x, y)=(2 n+1) x G_{n}(x, y)+\left(x y-2 x^{2}\right) \frac{\partial}{\partial x} G_{n}(x, y)+(2 x-x y) \frac{\partial}{\partial y} G_{n}(x, y) .
$$

The first few of the polynomials $G_{n}(x, y)$ are given as follows:

$$
G_{0}(x, y)=1, G_{1}(x, y)=x, G_{2}(x, y)=x y+x^{2}, G_{3}(x, y)=x y^{2}+4 x^{2} y+2 x^{2}+x^{3} .
$$

\subsection{Connection with Eulerian numbers}

Let $\mathfrak{S}_{n}$ denote the symmetric group of all permutations of $[n]$, where $[n]=\{1,2, \ldots, n\}$. Let $\pi=\pi(1) \pi(2) \ldots \pi(n) \in \mathfrak{S}_{n}$. A descent of $\pi$ is an index $i \in[n-1]$ such that $\pi(i)>\pi(i+1)$. Let des $(\pi)$ be the number of descents of $\pi$. The classical Eulerian polynomials are defined by

$$
A_{n}(x)=\sum_{\pi \in \mathfrak{S}_{n}} x^{\operatorname{des}(\pi)} .
$$

Let $\# C$ denote the cardinality of a set $C$. The Eulerian numbers are defined by

$$
\left\langle\begin{array}{l}
n \\
k
\end{array}\right\rangle=\#\left\{\pi \in \mathfrak{S}_{n}: \operatorname{des}(\pi)=k\right\} .
$$

Recall that the Eulerian numbers satisfy the recurrence relation

$$
\left\langle\begin{array}{c}
n+1 \\
k
\end{array}\right\rangle=(k+1)\left\langle\begin{array}{l}
n \\
k
\end{array}\right\rangle+(n+1-k)\left\langle\begin{array}{c}
n \\
k-1
\end{array}\right\rangle,
$$

with the initial conditions $\left\langle\begin{array}{l}1 \\ 0\end{array}\right\rangle=1$ and $\left\langle\begin{array}{l}1 \\ k\end{array}\right\rangle=0$ for $k \geqslant 1$. We can now present the following result.

Theorem 7. For $n \geqslant 1$ and $0 \leqslant k \leqslant n-1$, we have

$$
\gamma_{n, n-k, k}=\left\langle\begin{array}{l}
n \\
k
\end{array}\right\rangle \text {. }
$$

Proof. Set $a(n, k)=\gamma_{n, n-k, k}$. Then $a(n, k-1)=\gamma_{n, n-k+1, k-1}$. Using (8), it is easy to verify that $\gamma_{n, i, j}=0$ for $i+j>n$. Hence $\gamma_{n, n-k, k+1}=0$. Therefore, the numbers $a(n, k)$ satisfy the recurrence relation

$$
a(n+1, k)=(k+1) a(n, k)+(n+1-k) a(n, k-1) .
$$

Since the numbers $a(n, k)$ and $\left\langle\begin{array}{l}n \\ k\end{array}\right\rangle$ satisfy the same recurrence relation and initial conditions, they agree. 


\section{A bijective proof of Theorem 7:}

Proof. Let $\sigma \in \mathcal{Q}_{n}$. Note that every element of $[n]$ appears exactly two times in $\sigma$. Let $\alpha(\sigma)$ be the permutation of $\mathfrak{S}_{n}$ obtained from $\sigma$ by deleting each first occurrence of $i \in[n]$. Then $\alpha$ is a map from $\mathcal{Q}_{n}$ to $\mathfrak{S}_{n}$. For example, $\alpha(\mathbf{3 4 4 3 5 5 6 6 1 2 2 1 )}=435621$. Let

$$
\mathcal{D}_{n}=\left\{\sigma \in \mathcal{Q}_{n}: \operatorname{lap}(\sigma)=i, \operatorname{dasc}(\sigma)=n-i, \operatorname{dp}(\sigma)=0\right\} .
$$

It is clear that lap $(\sigma)+\operatorname{dasc}(\sigma)=n$ implies $\operatorname{dp}(\sigma)=0$ for $\sigma \in \mathcal{Q}_{n}$.

Let $x$ be a given element in $[n]$. For any $\sigma \in \mathcal{Q}_{n}$, we define an action $\beta_{x}$ on $\mathcal{Q}_{n}$ as follows:

- Read $\sigma$ from left to right and let $i$ be the first index such that $\sigma_{i}=x$;

- Move $\sigma_{i}$ to the right of $\sigma_{k}$, where $k=\max \left\{j \in\{0,1,2, \ldots, i-1\}: \sigma_{j}<\sigma_{i}\right\}$, where $\sigma_{0}=0$.

For example, if $\sigma=3443578876652211$, then

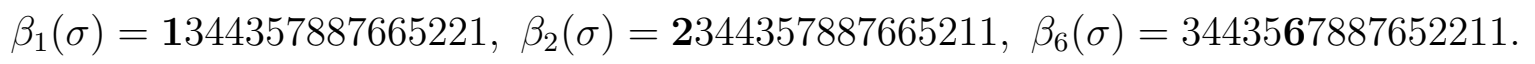

It is clear that $\beta_{x}\left(\beta_{y}(\sigma)\right)=\beta_{y}\left(\beta_{x}(\sigma)\right)$ for any $x, y \in[n]$. For any $S \subseteq[n]$, let $\beta_{S}: \mathcal{Q}_{n} \mapsto \mathcal{Q}_{n}$ be a function defined by

$$
\beta_{S}(\sigma)=\prod_{x \in S} \beta_{x}(\sigma)
$$

Clearly, $\beta_{[n]}(\sigma) \in \mathcal{D}_{n}$ and $\alpha(\sigma)=\alpha\left(\beta_{[n]}(\sigma)\right)$. Moreover, $\beta_{[n]}(\sigma)=\sigma$ if $\sigma \in \mathcal{D}_{n}$.

Let $\left.\alpha\right|_{\mathcal{D}_{n}}$ denote the restriction of the map $\alpha$ on the set $\mathcal{D}_{n}$. Then $\left.\alpha\right|_{\mathcal{D}_{n}}$ is a map from $\mathcal{D}_{n}$ to $\mathfrak{S}_{n}$. Let $\pi=\pi(1) \pi(2) \cdots \pi(n) \in \mathfrak{S}_{n}$. The inverse $\left.\alpha\right|_{\mathcal{D}_{n}} ^{-1}$ is defined as follows:

- let $\sigma=\sigma_{1} \sigma_{2} \ldots \sigma_{2 n}$ be the Stirling permutation such that $\sigma_{2 i-1}=\sigma_{2 i}=\pi(i)$ for each $i=1,2, \ldots, n$;

- let $S(\pi)=\left\{\pi_{i}: \pi_{i-1}>\pi_{i}, 2 \leqslant i \leqslant n\right\}$;

- let $\left.\alpha\right|_{\mathcal{D}_{n}} ^{-1}(\pi)=\beta_{S(\pi)}(\sigma)$.

Note that

$$
\operatorname{lap}\left(\left.\alpha\right|_{\mathcal{D}_{n}} ^{-1}(\pi)\right)+\operatorname{dasc}\left(\left.\alpha\right|_{\mathcal{D}_{n}} ^{-1}(\pi)\right)=n \text { and } \operatorname{dasc}\left(\left.\alpha\right|_{\mathcal{D}_{n}} ^{-1}(\pi)\right)=\operatorname{des}(\pi) .
$$

Then $\left.\alpha\right|_{\mathcal{D}_{n}}$ is a bijection from $\mathcal{D}_{n}$ to $\mathfrak{S}_{n}$. This completes the proof.

Example 8. The bijection between $\mathfrak{S}_{3}$ and $\mathcal{D}_{3}$ is demonstrated as follows:

$$
\begin{aligned}
& 123 \leftrightarrow 112233(S=\varnothing) \leftrightarrow \beta_{S}(112233)=112233 ; \\
& 132 \leftrightarrow 113322(S=\{2\}) \leftrightarrow \beta_{S}(113322)=112332 ; \\
& 213 \leftrightarrow 221133(S=\{1\}) \leftrightarrow \beta_{S}(221133)=122133 ; \\
& 231 \leftrightarrow 223311(S=\{1\}) \leftrightarrow \beta_{S}(223311)=122331 ; \\
& 312 \leftrightarrow 331122(S=\{1\}) \leftrightarrow \beta_{S}(331122)=133122 ; \\
& 321 \leftrightarrow 332211(S=\{1,2\}) \leftrightarrow \beta_{S}(332211)=123321 .
\end{aligned}
$$




\section{Concluding remarks}

In this paper, we introduce several variants of the ascent-plateau statistic on Stirling permutations. Here we provide another variant. Recall that the hyperoctahedral group $B_{n}$ is the group of signed permutations of the set $\pm[n]$ such that $\pi(-i)=-\pi(i)$ for all $i$, where $\pm[n]=\{ \pm 1, \pm 2, \ldots, \pm n\}$. As usual, we always identify a signed permutation $\pi=\pi(1) \cdots \pi(n)$ with the word $\pi(0) \pi(1) \cdots \pi(n)$, where $\pi(0)=0$. For each $\pi \in B_{n}$, we define

$$
\begin{aligned}
& \operatorname{des}_{A}(\pi)=\#\{i \in[n-1]: \pi(i)>\pi(i+1)\} \\
& \operatorname{des}_{B}(\pi)=\#\{i \in\{0,1,2 \ldots, n-1\}: \pi(i)>\pi(i+1)\} .
\end{aligned}
$$

Following [1], the number of flag descents of $\pi \in B_{n}$ is defined by

$$
\operatorname{fdes}(\pi)= \begin{cases}2 \operatorname{des}_{A}(\pi)+1, & \text { if } \pi(1)<0 \\ 2 \operatorname{des}_{A}(\pi), & \text { otherwise }\end{cases}
$$

Note that fdes $(\pi)=\operatorname{des}_{A}(\pi)+\operatorname{des}_{B}(\pi)$. In the same way, it is natural to introduce the definition of the number of flag ascent-plateaus. Let $\sigma=\sigma_{1} \sigma_{2} \cdots \sigma_{2 n} \in \mathcal{Q}_{n}$. The number of flag ascent-plateaus of $\sigma$ is defined by

$$
\operatorname{fap}(\sigma)= \begin{cases}2 \operatorname{ap}(\sigma)+1, & \text { if } \sigma_{1}=\sigma_{2} \\ 2 \operatorname{ap}(\sigma), & \text { otherwise }\end{cases}
$$

Clearly, $\operatorname{fap}(\sigma)=\operatorname{ap}(\sigma)+\operatorname{lap}(\sigma)$. A grammatical labeling of $\sigma \in \mathcal{Q}_{n}$ is given as follows:

$\left(L_{1}\right)$ If $i \in\{2,3, \ldots, 2 n-1\}$ is an ascent-plateau, then put a superscript label $y$ immediately before $\sigma_{i}$ and a superscript label $y$ right after $\sigma_{i}$;

$\left(L_{2}\right)$ If $\sigma_{1}=\sigma_{2}$, then put a superscript label $y$ immediately before $\sigma_{1}$ and a superscript $x$ right after $\sigma_{1}$;

$\left(L_{3}\right)$ If $\sigma_{1}<\sigma_{2}$, then put a superscript label $x$ immediately before $\sigma_{1}$;

$\left(L_{4}\right)$ The rest of positions in $\sigma$ are labeled by a superscript label $z$.

Note that the weight of $\sigma$ is given by $w(\sigma)=x y^{\mathrm{fap}(\sigma)} z^{2 n-\mathrm{fap}(\sigma)}$. It is routine to check that if $G=\left\{x \rightarrow x y z, y \rightarrow y z^{2}, z \rightarrow y^{2} z\right\}$, then

$$
D^{n}(x)=x \sum_{\sigma \in \mathcal{Q}_{n}} y^{\mathrm{fap}(\sigma)} z^{2 n-\mathrm{fap}(\sigma)} .
$$

Combining [12, Theorem 10], it is not hard to verify that

$$
\sum_{\pi \in B_{n}} x^{\mathrm{fdes}(\pi)+1}=\sum_{k=0}^{n}\left(\begin{array}{l}
n \\
k
\end{array}\right) \sum_{\sigma \in \mathcal{Q}_{k}} x^{\mathrm{fap}(\sigma)} \sum_{\rho \in \mathcal{Q}_{n-k}} x^{2 \operatorname{lap}(\rho)},
$$




$$
\sum_{\pi \in B_{n}} x^{\mathrm{fdes}(\pi)}=\sum_{k=0}^{n}\left(\begin{array}{l}
n \\
k
\end{array}\right) \sum_{\sigma \in \mathcal{Q}_{k}} x^{\mathrm{fap}(\sigma)} \sum_{\rho \in \mathcal{Q}_{n-k}} x^{2 \mathrm{ap}(\rho)} .
$$

In [18], Park studied the $(p, q)$-analogue of the descent polynomials of Stirling permutations:

$$
C_{n}(x, p, q)=\sum_{\sigma \in \mathcal{Q}_{n}} x^{\operatorname{des}(\sigma)} p^{\operatorname{inv}(\sigma)} q^{\operatorname{maj}(\sigma)}
$$

It would be interesting to provide a unified refinement of $C_{n}(x, p, q)$ and the following polynomials:

$$
\sum_{\sigma \in \mathcal{Q}_{n}} x^{\mathrm{ap}(\sigma)} y^{\operatorname{lap}(\sigma)} p^{\operatorname{inv}(\sigma)} q^{\operatorname{maj}(\sigma)}
$$

\section{Acknowledgements}

The authors thank the referee for his valuable suggestions which lead to a substantial improvement of the paper.

\section{References}

[1] R.M. Adin, F. Brenti, Y. Roichman. Descent numbers and major indices for the hyperoctahedral group. Adv. in Appl. Math., 27:210-224, 2001.

[2] M. Bóna. Real zeros and normal distribution for statistics on Stirling permutations defined by Gessel and Stanley. SIAM J. Discrete Math., 23:401-406, 2008/09.

[3] P. Brändén. Actions on permutations and unimodality of descent polynomials. European J. Combin., 29:514-531, 2008.

[4] W.Y.C. Chen. Context-free grammars, differential operators and formal power series. Theoret. Comput. Sci., 117:113-129, 1993.

[5] W.Y.C. Chen, A.M. Fu. Context-free grammars for permutations and increasing trees. Adv. in Appl. Math., 82:58-82, 2017.

[6] D. Foata, V. Strehl. Rearrangements of the symmetric group and enumerative properties of the tangent and secant numbers. Math. Z., 137:257-264, 1974.

[7] I. Gessel and R.P. Stanley. Stirling polynomials. J. Combin. Theory Ser. A, 24:25-33, 1978.

[8] J. Haglund, M. Visontai. Stable multivariate Eulerian polynomials and generalized Stirling permutations. European J. Combin., 33:477-487, 2012.

[9] S. Janson. Plane recursive trees, Stirling permutations and an urn model. Proceedings of Fifth Colloquium on Mathematics and Computer Science, Discrete Math. Theor. Comput. Sci. Proc., vol. AI:541-547, 2008. 
[10] S. Janson, M. Kuba and A. Panholzer. Generalized Stirling permutations, families of increasing trees and urn models. J. Combin. Theory Ser. A, 118: 94-114, 2011.

[11] Z. Lin, Jiang Zeng. The $\gamma$-positivity of basic Eulerian polynomials via group actions. J. Combin. Theory Ser. A, 135:112-129, 2015.

[12] S.-M. Ma. Some combinatorial arrays generated by context-free grammars. European J. Combin., 34:1081-1091, 2013.

[13] S.-M. Ma. A family of two-variable derivative polynomials for tangent and secant. Electron. J. Combin., 20(1):\#P11, 2013.

[14] S.-M. Ma, T. Mansour. The $1 / k$-Eulerian polynomials and $k$-Stirling permutations. Discrete Math., 338:1468-1472, 2015.

[15] S.-M. Ma, T. Mansour, D.G.L. Wang, Y.-N. Yeh. Several variants of the Dumont differential system and permutation statistics. Sci. China Math., (2018). doi:10.1007/s11425-016-9240-5

[16] S.-M. Ma, Y.-N. Yeh. Stirling permutations, cycle structure of permutations and perfect matchings. Electron. J. Combin., 22(4):\#P4.42, 2015.

[17] S.-M. Ma, Y.-N. Yeh. Eulerian polynomials, Stirling permutations of the second kind and perfect matchings. Electron. J. Combin., 24(4):\#P4.27, 2017.

[18] S.K. Park. The r-multipermutations. J. Combin. Theory Ser. A, 67(1):44-71, 1994. 